\title{
PRODUCTION PRIMAIRE ET BILAN DE L'OXYGENE DISSOUS DANS UN RUISSEAU DES PYRẼNEES CENTRALES
}

\author{
par J. CapblancQ ${ }^{1}$ et P. Lavandier ${ }^{1}$.
}

\begin{abstract}
La méthode amont-aval est utilisée pour estimer la production primaire d'un petit ruisseau de moyenne altitude. Les processus de décomposition excèdent largement la production primaire brute. La réduction de l'éclairement par la végétation arbustive des rives, le fort apport de matières organiques lors de la chute automnale des feuilles et l'envasement progressif lié à l'absence de crues apparaissent comme les principaux facteurs responsables du métabolisme de cet écosystème.

Même dans des conditions d'utilisation limites (faibles variations de l'oxygène dissous, forte diffusion, temps de rétention court, apports par drainage) la méthode amont-aval peut fournir des estimations de production valables; les principales causes d'erreur sont analysées.
\end{abstract}

\section{Primary production and oxygen budget in a stream of the Central Pyrenees.}

The method of comparing changes in the dissolved oxygen concentration between upstream and downstream stations was used to estimate primary production in a small stream at a mean altitude in the Pyrenees. Decomposition processes greatly exceeded gross primary production. The reduction in illumination by the shrubs on the bank, the large input of organic material at the time of the autumnal fall of leaves, and the progressive silting coupled with the absence of floods appeared to be the chief factors responsible for the metabolism of this ecosystem.

In spite of some limiting factors (low variations in dissolved oxygen, strong diffusion, short retention time, input through drainage), the upstream-downstream comparative method can provide some valid estimates of production. The principal causes of error are analysed.

L'utilisation des variations journalières d'oxygène dissous pour calculer la production primaire des caux courantes fut introduite par Odum (1956 et 1957 a et b). Cette méthode permet de mesurer le métabolisme des communautés dans les conditions naturelles. Elle n'est cependant applicable qu'à des milieux suffisamment calmes pour que les phénomènes d'aération ne masquent pas les manifestations du métabolisme. Par suite, les mesures de production primaire réalisées en eau courante par la méthode amont-

1. Laboratoire d'Hydrobiologie, Université Paul-Sabatier, 118, route de Narbonne, 31077 Toulouse Cedex, France. 
aval restent peu nombreuses (Hoskin 1959, Edwards et Cwens 1962, Owens 1965, Duffer et Doris 1966, Hall 1972, McDiffet et al. 1972).

Le présent travail a pour but d'une part de dresser le bilan en oxygène dissous d'un petit ruisseau de moyenne altitude par la méthode amont-aval, d'autre part de tester la sensibilité de cette méthode en l'appliquant à un milieu de dimensions réduites, à faibles variations journalières de l'oxygène dissous et à forte diffusion.

\section{1. — Le milieu.}

Le ruisseau étudié est situé dans les Pyrénées centrales à $780 \mathrm{~m}$ d'altitude. Il coule au travers d'une aulnaie dans les alluvions de la Neste d'Aure où il se jette après un cours de 330 mètres (fig. 1).

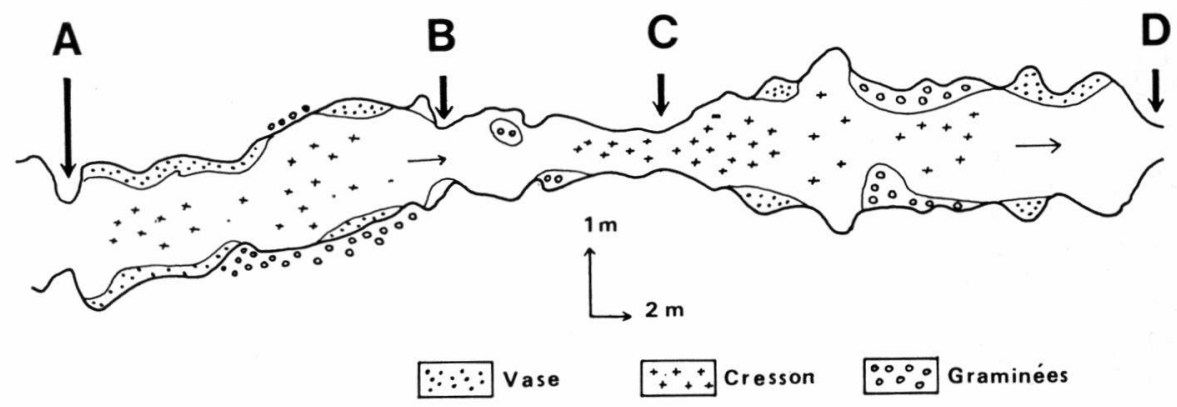

Fig. 1. - Milieu étudié et stations d'étude.

Alimenté par la nappe phréatique qu'entretient la Neste dans les alluvions, son débit peut présenter des variations saisonnières non négligeables : fonction du niveau de la nappe le débit est de 50 à $80 \%$ plus élevé à la fonte des neiges qu'à l'étiage. Il est par contre relativement constant sur de courtes durées et, pour une saison donnée, d'une année à l'autre : sur 15 mesures réalisées d'avril 1971 à avril 1975 les valeurs extrêmes furent de 13,8 à $21,5 \mathrm{l} / \mathrm{s}$ à la station C. Du fait de l'apport de sources diffuses, le débit augmente progressivement de l'amont vers l'aval ; cette augmentation s'effectue dans les mêmes proportions quelle que soit la saison. A la station A le débit est égal à $84 \% \pm 1,5 \%$ du débit en $\mathrm{C}$; à la station $\mathrm{D}$ il est de $125 \% \pm 2 \%$.

Ce ruisseau présente une alternance de zones étroites (1,5 à $2 \mathrm{~m}$ ) et peu profondes (5 à $10 \mathrm{~cm}$ ) — zone $\mathrm{BC}$ — et de biefs plus larges $(2$ à $3 \mathrm{~m})$ et plus profonds $(10-25 \mathrm{~cm})$ - zones $\mathrm{AB}$ et CD - Dans les premières, le substrat de galets est partiellement recouvert de mousses (Fontinalis antipyretica). Dans les 
secondes, le fond sableux est presque uniformément couvert d'une couche de vase fine. Le cresson des fontaines (Nasturtium officinale) occupe 60 à $75 \%$ du fond et ralentit le courant. Au début du printemps (mars) le ruisseau est envahi par des chlorophycées filamenteuses (Mougeotia $s p$. et Spirogyra sp.) qui s'accrochent sur les tiges de cresson et servent de support à des diatomées où dominent Diatoma vulgare, Synedra acus, Cocconeis placentula, Achnanthes minutissima. Ce périphyton disparaît dès la fin du printemps tandis qu'augmente l'ombrage de l'aulnaic.

La biomasse végétale moyenne est significativement plus importante au début du printemps $\left(79 \mathrm{~g} \mathrm{PS} / \mathrm{m}^{2}\right.$ - limites de confiance à $\mathrm{P} 0,05: 66$ à $94 \mathrm{~g} \mathrm{PS} / \mathrm{m}^{2}$ ) qu'en juillet et en septembre où elle est respectivement de $41,5(24,5$ à 70$)$ et de $26(15,5$ à 36$) \mathrm{g} \mathrm{PS} / \mathrm{m}^{2}$. En été, la température de l'eau excède rarement $12{ }^{\circ} \mathrm{C}$ et ses variations journalières ne dépassent pas $0,5^{\circ} \mathrm{C}$. En mars-avril, le ruisseau étant peu ombragé, la température varie de $6{ }^{\circ} \mathrm{C}$ la nuit à $10,5^{\circ} \mathrm{C}$ le jour.

L'eau du ruisseau a une composition chimique assez constante. Son $\mathrm{pH}$ est compris entre 7,5 et 7,85 ; sa conductivité à $18^{\circ} \mathrm{C}$ est de $230 \mu$ mhos, l'alcalinité totale de $2,2 \mathrm{méq} / \mathrm{l}$.

\section{2. — Méthodes.}

Entre deux stations successives la différence $\mathrm{X}$ des teneurs en oxygène dissous résulte de l'action conjuguée de plusieurs facteurs :

$\mathrm{X}=$ Photosynthèse - Respiration \pm Diffusion + Apports de drainage. Si on connaît le temps moyen (1) nécessaire à l'eau pour parcourir la distance séparant les deux stalions, le bilan exprimé par $\mathrm{m}^{2}$ de ruisseau sera :

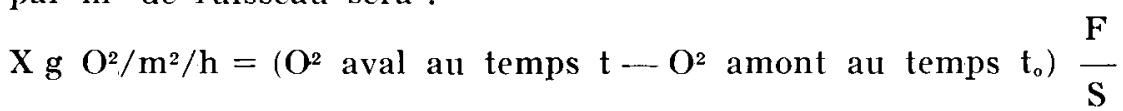
où $\mathrm{F}=$ débit en $\mathrm{m}^{3} / \mathrm{h}$ et $\mathrm{S}=$ superficie du ruisseau entre les stations en $\mathrm{m}^{2}$.

Nous avons calculé le débit en amont et en aval des biefs étudiés par intégration de mesures faites au micromoulinet. Un appareil enregistreur de niveau (Lavandier 1974) a été placé dans le ruisseau pour déterminer les variations éventuelles du débit pendant l'expérience.

La largeur et la profondeur moyenne de chaque bief ont été déterminées à partir de mesures transversales faites tous les mètres. Le temps de rétention ( $t$ ) peut ainsi être calculé à partir des paramètres morphodynamiques du bief considéré $(t=$ volume du bief/débit moyen). Dans les biefs étudiés cette valeur calculéc diffère très peu des valeurs obtenues en mesurant le temps de 
parcours de traceurs (fluorescéine, vague de désoxygénation) entre les stations de mesure.

Nous avons dosé l'oxygène dissous par la méthode de Winkler toutes les heures à la station d'amont, toutes les heures plus t minutes aux stations d'aval. Deux dosages ont été réalisés à chaque relevé.

La différence de débit entre les stations d'amont et d'aval correspond à une entrée d'eau de la nappe dans le bief étudié. La teneur en oxygène dissous de cette eau, dosée dans le ruisseau aux résurgences de la nappe est assez constante au cours de l'année et égale à $4 \pm 0,45 \mathrm{ppm}(\mathrm{P} 0,05)$.

L'estimation de la diffusion requiert le calcul du coefficient de diffusion f : celui-ci peut être établi à partir de l'évolution du déficit nocturne en $\mathrm{O}^{2}$. Ce déficit variant peu au cours de la nuit, nous avons provoqué une chute de la tension en $\mathrm{O}^{2}$ en ajoutant

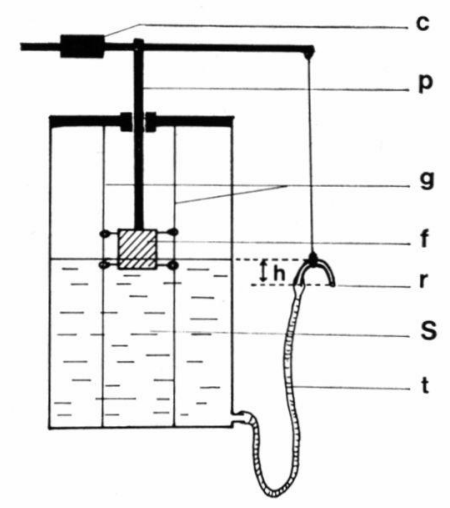

FIG. 2. - Appareil à débit constant utilisé. L’appareil comprend : un container de 50 litres, un flotteur (f) coulissant le long de 2 guides verticaux (g), une potence (p) munie d'un contrepoids (c). Fixée au flotteur la potence supporte un tuyau de vidange souple relié à la base du container.

Au fur et à mesure que le liquide (s) s'écoule le flotteur descend, entrâ̂nant le système de vidange qui lui est solidai e. Ainsi la hauteur (h) entre la surface du liquide et l'orifice d'écoulement (r) reste constante.

une solution de sulfite de sodium avec du nitrate de cobalt comme catalyseur, selon une méthode utilisée par Gameson et al. (1955) et Edwards et al. (1961). Le déversement de cette solution en amont de la zone étudiée est réalisé grâce à un appareil à débit constant de conception simple, décrit dans la figure 2. La variation des teneurs en oxygène dissous entre les stations de mesure avant et pendant le passage de l'eau partiellement désoxygénée (fig. 3) permet de calculer le coefficient de diffusion (f). 

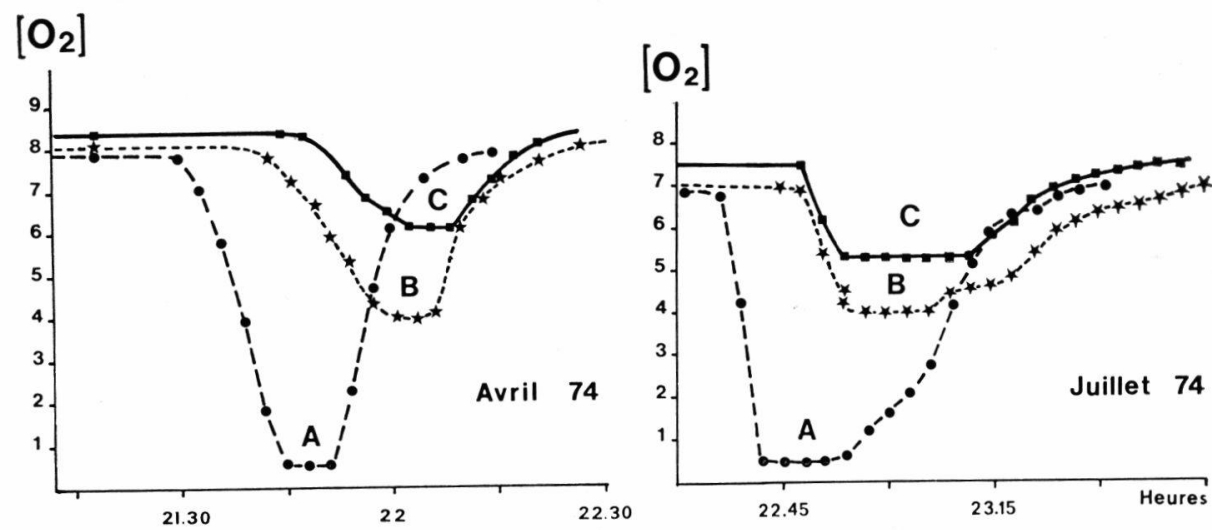

Fig. 3. - Expérience de désoxygénation au sulfite : évolution des teneurs en $\mathrm{O}$, dissous aux stations $\mathrm{A}, \mathrm{B}$, et $\mathrm{C}$.

Connaissant la diffusion (D) et l'apport d'oxygène par drainage, on déduit le taux de respiration $(R)$ des valeurs nocturnes de $X$. Afin de corriger $\mathbf{R}$ en fonction des températures diurnes nous avons mesuré, lors d'expériences in vitro, le taux de consommation d'oxygène des végétaux et de la vase aux températures extrêmes du ruisseau - les échantillons prélevés dans le ruisseau étant placés dans des flacons de DBO, avec agitation de l'eau.

\section{3. - Résultats .}

De 1971 à 1975, nous avons réalisé 11 séries de mesures : 6 au printemps (fin mars - début avril), 5 en été (juillet et septembre). Les résultats sont récapitulés dans le tableau I. Dans les biefs étudiés, l'eau reste constamment sous-saturée en oxygène dissous. La variation d'oxygène est faible entre les stations A et B ; l'oxygénation est plus importante dans les biefs B-C à courant plus rapide (fig. 4). L'amplitude des variations nuit - jour atteint $1 \mathrm{ppm}$ en avril ; elle est inférieure à $0,5 \mathrm{ppm}$ en juillet et septembre.

Dans ce milieu sous-saturé et peu profond, la diffusion constitue l'apport essentiel d'oxygène dissous quelle que soit la saison. Le coefficient d'échange $(\mathrm{f} \mathrm{cm} / \mathrm{h}$ ) est relativement élevé du fait de la faible épaisseur de la lame d'eau. Il existe une corrélation hautement significative entre les valeurs de $f$ calculées dans les différents biefs et la vitesse moyenne du courant de ces biefs $(\overline{\mathrm{V}}=$ longueur du bief/temps de rétention) (fig. 5). Les valeurs de f mesurées par la méthode au sulfite sont comparables à celles que donne l'équation de régression établie par Owens et al. (1964) 

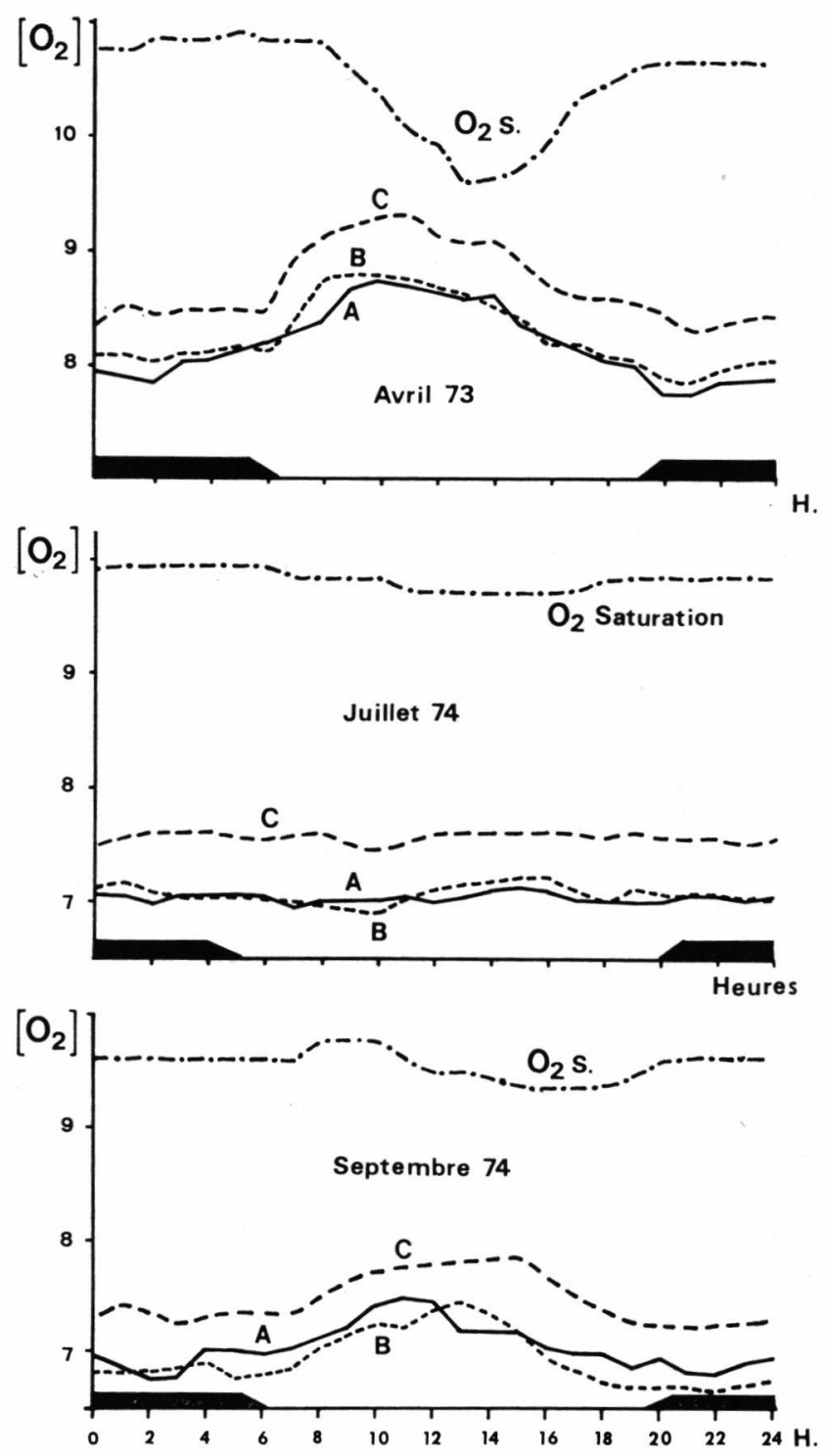

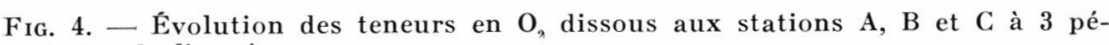
riodes de l'année.

$\left[\mathrm{f}_{20}=50,8 \cdot \mathrm{V}^{0,67} \cdot \mathrm{p}^{-0,85}\right]$ après correction pour la température par la relation $\mathrm{f}_{\mathrm{T}}^{\circ}=\mathrm{f}_{20}{ }^{\circ} \cdot 1,024\left(\mathrm{t}^{\circ}-20\right) \quad$ (Churchill et al. 1962) (fig. 6). L'apport d'oxygène par diffusion représente ainsi 66 à $95 \%$ des gains journaliers dans les zones à courant lent (A - B et 


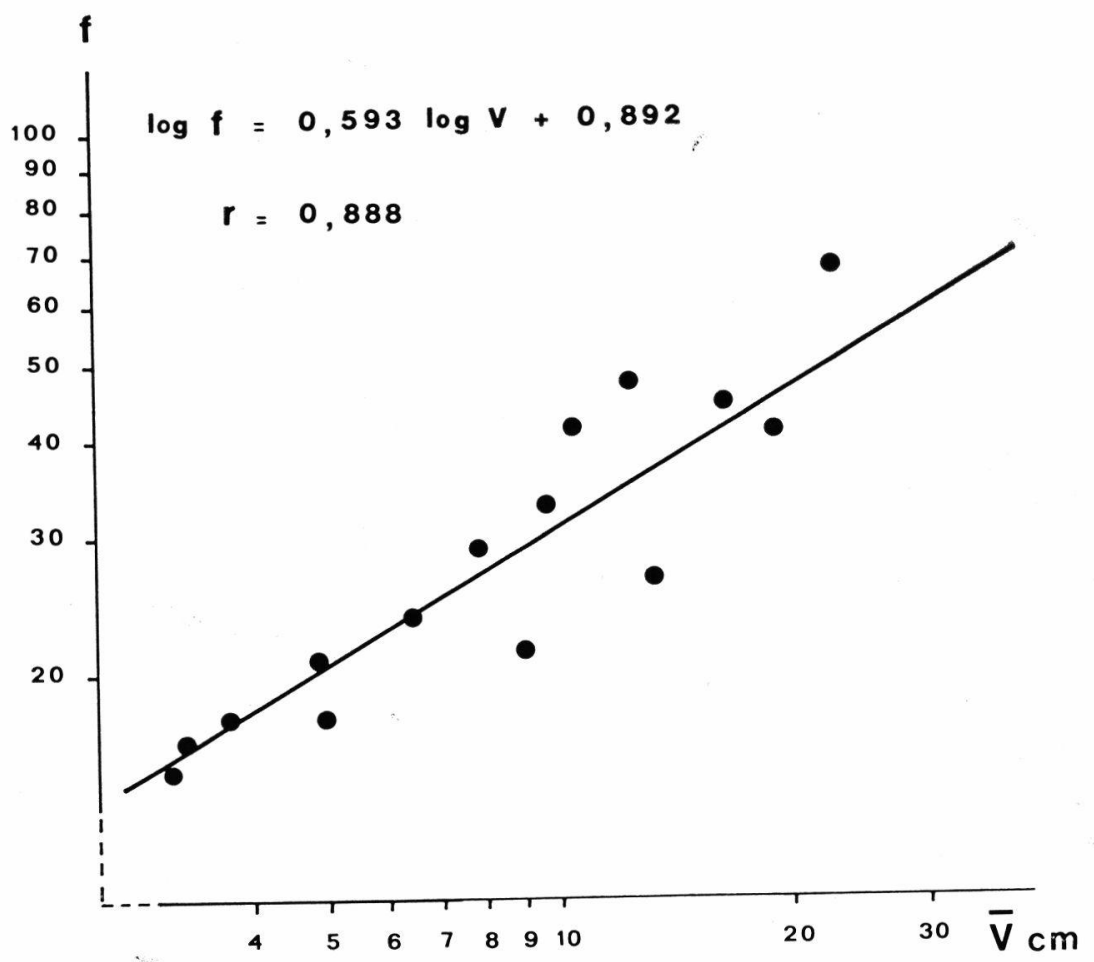

Fig. 5. - Relations entre la vitesse moyenne du courant et le coefficient d'échange $f$.

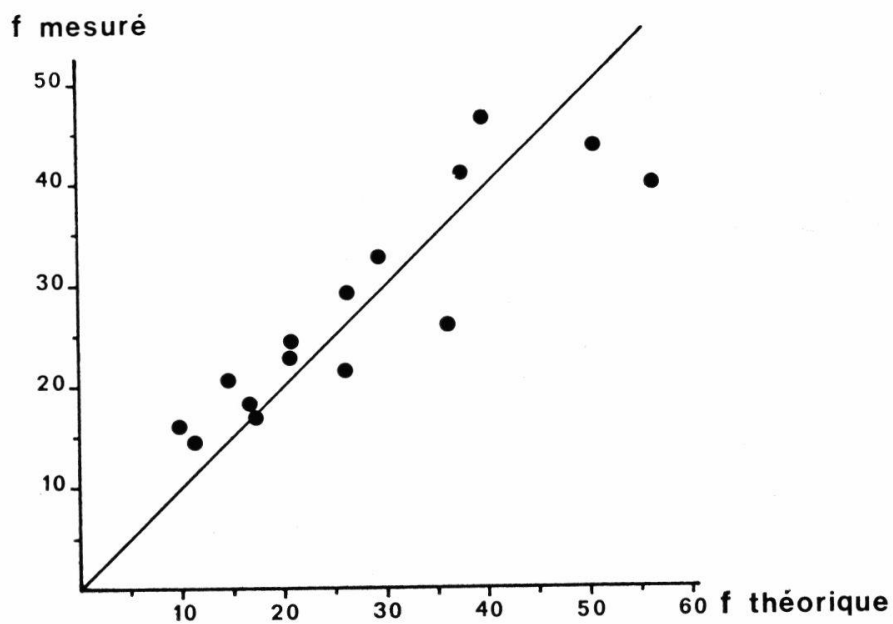

Fig. 6. - Comparaison entre les valeurs du coefficient d'échange $f$ mesurées et celles calculées d'après la relation $\mathrm{f}_{20^{\circ}}=50,8 . \mathrm{V}^{0},{ }^{67} . \mathrm{P}^{-0},{ }^{85}$. 
C - D), $78 \%$ à $97 \%$ dans les zones à courant plus rapide $(\mathrm{B}-\mathrm{C})$. Dans la zone A-C $30 \%$ (juillet) à $50 \%$ (avril) de cet apport sont nécessaire pour combler la différence de concentration en oxygène dissous existant entre l'eau de la nappe et celle du ruisseau.

Le taux de respiration nocturne varie de $0,13 \mathrm{~g} \mathrm{O}^{2} / \mathrm{m}^{2} / \mathrm{h}$ à $0,61 \mathrm{~g} \mathrm{O}^{2} / \mathrm{m}^{2} / \mathrm{h}$. D'une manière générale, il est plus faible en mars - avril où sa valeur moyenne à $\mathrm{P} 0,05$ est de $0,193 \pm 0,055 \mathrm{~g}$ $\mathrm{O}^{2} / \mathrm{m}^{2} / \mathrm{h}$ qu'en juillet et septembre $\left(0,364 \pm 0,22 \mathrm{~g} \mathrm{O}^{2} / \mathrm{m}^{2} / \mathrm{h}\right)$.

\begin{tabular}{|c|c|c|c|c|c|}
\hline Date & Section & g $\frac{D}{O_{2} / m^{2} / j}$ & $\underset{\mathrm{g} \mathrm{O}_{\mathrm{z}} / \mathrm{m}^{2} / \mathrm{j}}{\mathrm{R}}$ & $\underset{\mathrm{g} \mathrm{O}_{2} / \mathrm{m}^{2} / \mathrm{j}}{\mathbf{P}}$ & $\mathrm{P} / \mathrm{R}$ \\
\hline 20- IV-71 & A-B & 10,57 & 5,90 & 2,25 & 0,38 \\
\hline 12- IV-72 & A-B & 8,61 & 6,79 & 2,80 & 0,41 \\
\hline 10- IV-73 & A-B & $\mathbf{7 , 7 7}$ & 4,77 & 2,43 & 0,51 \\
\hline 10- IV-73 & B-C & 13,67 & 4,27 & 1,46 & 0,34 \\
\hline 3- IX-73 & $\bar{A}-\bar{B}$ & 8,50 & 5,22 & 0,77 & 0,15 \\
\hline 25- III-74 & A-B & 8,24 & 3,63 & 3,56 & 0,98 \\
\hline 2-VII-74 & A-B & 19,80 & 14,71 & 0.77 & 0,05 \\
\hline 2-VII-74 & B-C & 32,47 & 4,21 & 0,34 & 0,08 \\
\hline 2- IX-74 & A-B & 9,92 & 8,85 & 1,28 & 0,14 \\
\hline $22-$ III-75 & C-D & 6,12 & 5,88 & 2,81 & 0,48 \\
\hline 3-VII-75 & C-D & 17,77 & 10,75 & 1,56 & 0,09 \\
\hline
\end{tabular}

TableaU I. - Diffusion, respiration et production brute dans trois sections du ruisseau.

Les mesures réalisées in vitro ont donné des taux de consommation figurés dans le tableau II. La "respiration》 du cresson augmente plus vite avec la température $(Q 10=5,8)$ que celle de la vase $(\mathrm{Q} 10=2,9)$. La consommation $\mathrm{d}^{\prime} \mathrm{O}^{2}$ par le cresson à $10,5^{\circ} \mathrm{C}$ est comparable à celles mesurées à $10^{\circ} \mathrm{C}$ par Owens et Marris (1964) pour quatre espèces de végétaux aquatiques $(0,8$ à $1 \mathrm{mg} \mathrm{O}^{2} / \mathrm{g} \mathrm{PS} / \mathrm{h}$ pour des teneurs en $\mathrm{O}^{2}$ de 7 à $\left.8 \mathrm{ppm}\right)$. Ces auteurs montrent que la valeur du $Q 10$ varie selon les espèces entre 3,48 et 1,4 pour des températures comprises entre $10^{\circ} \mathrm{C}$ et $15{ }^{\circ} \mathrm{C}$. Par contre la consommation en oxygène de la vase du ruisseau est plus faible que celle donnée par Edwards et Rolley (1965) qui ont mesuré des consommations de 0,6 à $0,7 \mathrm{mg}$ $\mathrm{O}^{2} / 100 \mathrm{~cm}^{2} / \mathrm{h}$ à $10{ }^{\circ} \mathrm{C}$.

Appliqués au ruisseau, les taux de consommation mesurés in vitro donnent une valeur moyenne de respiration de $0,052(0,022$ à 0,089$) \mathrm{g} \mathrm{O}^{2} / \mathrm{m}^{2} / \mathrm{h}$ en mars - avril et de $0,061(0,03$ à 0,124$) \mathrm{g}$ $\mathrm{O}^{2} / \mathrm{m}^{2} / \mathrm{h}$ en juillet et septembre. Les résultats sont $3, \bar{\imath}$ à 6 fois plus faibles que ceux estimés par la méthode amont-aval ; cette différence est certainement due en partie à une importante sousestimation de la consommation d'oxygène par la vase établie in vitro. 


\begin{tabular}{|c|c|c|}
\hline \multirow[b]{2}{*}{ Substrat } & \multicolumn{2}{|c|}{ Température } \\
\hline & $\begin{array}{c}6^{\circ} 5 \\
\overline{\mathrm{X}} \pm \mathrm{I}_{1} . \mathrm{C}_{0.05}\end{array}$ & $\begin{array}{c}10^{\circ} 5 \\
\overline{\mathrm{X}} \pm \mathrm{L} \cdot \mathrm{C}_{0,0:}\end{array}$ \\
\hline $\begin{array}{l}\text { Cresson } \\
\mathrm{mg} \mathrm{O} \mathrm{O}_{\mathrm{g}} / \mathrm{g} \text { Ps } / \mathrm{h}\end{array}$ & $0,37 \pm 0,19$ & $0,75 \pm 0,26$ \\
\hline $\begin{array}{l}\text { Vase } \\
\mathrm{mg} \mathrm{O}_{2} / 100 \mathrm{~cm}^{2} / \mathrm{h}\end{array}$ & $0,23 \pm 0,13$ & $0,37 \pm 0,16$ \\
\hline
\end{tabular}

Tableau II. - Taux de consommation d'oxygène du cresson et de la vase établis lors de mesures in vitro.

Le calcul de la respiration diurne a été fait en admettant un $\mathrm{Q} 10$ moyen de 4,3 , valeur très proche de celle qui peut se déduire de la courbe de Krogh (in Winberg 1971) entre 5 et $10^{\circ} \mathrm{C}$. La consommation journalière moyenne d'oxygène par les communautés benthiques est plus élevée en été $\left(8,75 \pm 5,29 \mathrm{~g} \mathrm{O}^{2} / \mathrm{m}^{2} / \mathrm{j}\right)$ qu'au printemps $\left(5,2 \pm 1,24 \mathrm{~g} \mathrm{O} \mathrm{O}^{2} / \mathrm{m}^{2} / \mathrm{j}\right)$. Inversement la production primaire brute est plus forte au début du printemps $(2,55 \pm 0,73 \mathrm{~g}$ $\left.\mathrm{O}^{2} / \mathrm{m}^{2} / \mathrm{j}\right)$ que durant l'été $\left(0,94 \pm 0,6 \mathrm{~g} \mathrm{O}^{2} / \mathrm{m}^{2} / \mathrm{j}\right)$.

Cette évolution correspond aux changements observés dans les caractéristiques thermiques, la végétation du ruisseau (invasion par les algues au printemps) et celle des rives (réduction de l'éclairement par le feuillage de l'aulnaie à partir de la fin avril).

\section{4. - Discussion.}

La production primaire brute du ruisseau étudié est parmi les plus faibles citées dans la littérature. Si Hall (1972) indique une production primaire moyenne de $0,8 \mathrm{~g} \mathrm{O}^{2} / \mathrm{m}^{2} / \mathrm{j}$ avec des valeurs extrêmes de 0,2 à $9 \mathrm{~g} \mathrm{O}^{2} / \mathrm{m}^{2} / \mathrm{j}$ dans une rivière de Caroline du Nord, les productions mesurées par la méthode amont-aval se situent plus généralement entre 5,62 (McDiffet et al. 1972) et 20 (Duffer et Doris 1966) $\mathrm{g} \mathrm{O}^{2} / \mathrm{m}^{2} / \mathrm{j}$.

Le rapport $\mathrm{P} / \mathrm{R}$ constitue, selon Odum (1956) un bon indicateur de l'état trophique de l'écosystème. Dans le ruisseau étudié, il varie entre $1 / 3$ et 1 en mars - avril, entre $1 / 20$ et $1 / 7$ en été (tableau I). Ce milieu constitue donc un écosystème hétérotrophe présentant une légère tendance à l'autotrophie au début du printemps, période de développement d'un «bloom» algal. La faiblesse de la production primaire endogène en regàrd des processus de décomposition est attribuable à l'envasement progressif du ruisseau et à la réduction de l'éclairement par la couverture arbustive des rives; elle traduit l'importance des apports de matière organique exogènes. 
Toutefois, pour un écosystème, la signification du rapport $P / R$ diffère selon l'importance relative des entrées et des sorties de matic̀res organiques (Fisher et Likens 1973). Dans ce ruisseau, l'apport exogène de matières organiques est essentiellement déterminé par la chute automnale des feuilles et par les substances dissoutes au contact du sol dans l'eau de la nappe. Du fait de l'absence de crues et de l'encombrement du lit, les feuilles mortes tendent à s'accumuler dans les zones à courant lent. Les substances dissoutes dégradables sont sans doute présentes en faible quantité puisque l'incubation d'échantillons d'eau en flacons DBO n'a révélé que des consommations d'oxygène nulles ou négligeables. Les matériaux transportés vers l'aval sont composés essentiellement de débris végétaux; la filtration de l'eau au travers de filets à vide de maille de $150 \mu$ placés en divers points du ruisseau a montré que la quantité de débris organiques transportés varie de 10 à $35 \mathrm{~g}$ de poids sec/24 heures.

Dans les diverses sections du ruisseau, les valeurs de $\mathbf{P}$ et de $\mathbf{R}$ ne diffèrent pas notablement ef les résultats obtenus à des périodes identiques indiquent une certaine stabilité du cycle saisonnier. Le développement printanier des algues vertes, lié à l'éclairement, détermine un pic de production annuel. Après la disparition de ces algues au début de l'été, la production primaire par lc cresson reste faible tandis que les processus de dégradation, consommateurs d'oxygène, augmentent avec la température moyenne du ruisseau.

La méthode amont-aval utilisée pour évaluer le métabolisme des communautés d'eaux courantes est soumise à de multiples causes d'erreurs. L'analyse des résultats obtenus sur ce ruisseau montre que les paramètres suivants sont suceptibles de modifier de façon sensible les résultats finaux :

- le débit

- la diffusion

- les variations journalic̀res de $\mathbf{R}$

- l'apport par drainage.

Les diverses méthodes existant pour tarer un cours d'eau fournissent une estimation du débit avec une marge d'erreur rarement inférieure à $10 \%$. Cette erreur se traduit par une erreur relative identique dans l'expression du coefficient d'échange (f), de la respiration $(R)$ et de la photosynthèse $(P)$.

La diffusion (D) est sans doute un des paramètres le plus difficile à mesurer. Elle est fonction du déficit (d) en $\mathrm{O}^{2}$ par rapport à la saturation et du coefficient d'échange (f). Son calcul repose donc sur l'hypothèse que : 
- dans un bief, D est proportionnel à la moyenne arithmétique du déficit en $\mathrm{O}^{2}$ aux stations amont et aval :

$$
\mathrm{D}=\frac{\mathrm{f}}{100}\left(\frac{\mathrm{d} \text { amont }+\mathrm{d} \text { aval }}{2}\right)
$$

- R est indépendant de la teneur en $\mathrm{O}^{2}$ et, en particulier, qu'il reste constant pendant l'expérience de désoxygénation partielle au sulfite ;

- les conditions extérieures (vent, variations de pression atmosphérique) ne modifient pas la valeur de f au cours d'un cycle de $24 \mathrm{~h}$;

-- le mélange longitudinal de l'eau est homogène dans le bief étudié.

Toute variation de $f$ entraîne une erreur dans l'estimation du taux horaire de $\mathbf{R}$ proportionnelle au déficit nocturne (dn). De même, l'erreur sur $P$ est proportionnelle à la différence entre le déficit nocturne $(d n)$ et le déficit diurne $(d j)$, c'est-à-dire qu'une variation de $f$ entraîne une erreur sur $P$ d'autant plus importante que le cycle nycthéméral des teneurs en oxygène dissous est accusé.

La respiration $(R)$ varie en fonclion de la température et de la tension en $\mathrm{O}^{2}$ (Owens et Marris 1964). La tension en $\mathrm{O}^{2}$ restant à peu près constante dans le ruisseau étudié, seules les variations de température sont à prendre en considération. La correction apportée pour les variations de température en mars - avril affecte le résultat final, $\mathrm{P}$ et $\mathrm{P}$ étant augmentés respectivement de $12 \%$ et de $28,4 \%$ en moyenne par rapport aux valeurs calculées pour $\mathrm{R}$ constant.

L'apport d'eau de drainage, faiblement oxygénée, représente une part importante du débit du ruisscau dans les biefs étudiés el, pour un bief donné, une fraction constante du débit. La teneur en oxygène de cette eau de nappe varie peu au cours de l'année. Quelle que soit la valeur affectée à cette teneur, les valeurs de f et de $P$ ne sont pas modifiées. Par contre, l'estimation de $R$ est différente selon la concentration en $\mathrm{O}^{2}$ dissous attribuée à l'eau de drainage. D'une manière générale, une surélévation de $1 \mathrm{ppm}$ de la quantité de $\mathrm{O}^{2}$ apportée par drainage détermine une surévaluation du taux de respiration horaire d'une valeur égale au rapport débit de la nappe/superficie de la section.

Nous récapitulons dans le tableau III les causes d'erreur susceptibles de modifier nos résultats et les valeurs de $P$ et $R$ correspondantes. Les marges d'erreur concernant les diverses variables sont fixées de façon approximative et, d'après notre expérience, représentent sans doute l'erreur maximum envisageable. Ces résultats montrent que la production peut être estimée avec une erreur probablement inférieure à $25 \%$ si le débit du ruisseau étudié et 


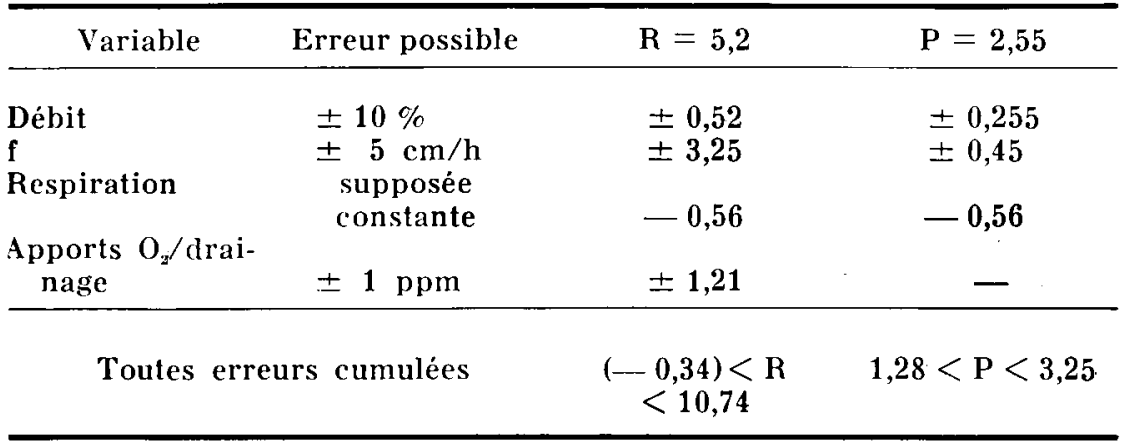

Tableau III. - Evaluation de l'erreur maximale susceptible d'être commise sur les mesures. Chaque paramètre est affecté de la plus forte erreur d'estimation envisageable. $P$ et $R$ sont exprimés en $\mathrm{g} \mathrm{O}_{\mathrm{z}} / \mathrm{m}^{2} /$ jour.

le coefficient d'échange sont mesurés avec précaution. L'erreur diminuera d'autant plus que f sera faible (ruisseaux lents et assez profonds) et que les variations journalières des teneurs en $\mathrm{O}^{2}$ seront peu prononcées.

Dans notre étude, il est par contre plus difficile d'obtenir une valeur précise de $R$. Le débit et la concentration en $\mathrm{O}^{2}$ de l'eau de drainage déterminent un déficit important dans l'cau du ruisseau si bien que toule crreur sur f entraîne une forte erreur sur R. Dans les ruisseaux à forte activité métabolique, le déficit nocturne est parfois plus accusé; mais le coefficient de diffusion étant généralement plus faible, l'estimation de $f$ influe moins sur celle de $R$. Si les conditions hydrodynamiques réunies ici ne semblent pas être le cas général, il est toutefois probable que tout ruisseau reçoit à partir de la nappe ou du sous-écoulement une eau moins oxygénée. Ne pas tenir compte de cet apport revien! à surévaluer $R$ de façon d'autant plus importante que le débit de la nappe est proportionnellement élevé, c'est-à-dire que l'on se trouve en période d'étiage.

\section{TRAVAUX CITÉS}

Churchill (M. A.), Eldmore (H. L.) et Buckingham (R. A.). 1962. - The prediction of stream reaeration rates. Int. J. Air Wat. Pollut., 6 : 467-504.

Duffer (W. R.) et Doris (T. C.). 1966. - Primary productivity in a southern great plains stream. Limnol. Océanogr., 11 : 143-151.

Edwards (R. W.), Owens (M.) et Girbs (J. W.). 1961. - Estimates of surface acration in two streams. J. Inst. Wat. Engrs., $15: 395-405$.

EDwards (R. W.) et Owens (M.). 1962. - The effect of plants on river conditions. IV. The oxygen balance of a chalk stream. J. Ecol., $50: 207-220$. 
Edwards (R. W.) et Rolley (H. L. J.), 1965. - Oxygen consumption of river muds. J. Ecol., $53: 1-19$.

Fisher (S. G.) et Likens (G. E.). 1973. - Energy flow in Bearbrook, New Hampshire: an integrative approach to stream ecosystem metabolism. Ecol. Monogr., 43 (4) : 421-439.

Gameson (A. L. H.), Truesdale (G. A.) et Downing (A. L.). 1955. Reaeration studies in a lakeland beck. J. Inst. Wat. Engrs., 9 : $57-94$.

HaLl (C. A. S.). 1972. - Migration and metabolism in a temperate stream ecosystem. Ecology, 53 (4) : 585-605.

Hoskin (C. M.). 1959. - Studies of oxygen metabolism of streams in North Carolina. Publ. Inst. Mar. Sci. Univ. Texas, 6 : 186-192.

Lavandier (P.). 1974. - Écologie d'un torrent pyrénéen de haute montagne. I. Caractéristiques physiques. Annls. Limnol., 10 (2) : 173219.

McDiffet (W. F.), Carr (A. E.) et Young (D. L.). 1972. -- An estimate of primary prorluctivity in a Pensylvania trout stream using a diurnal oxygen curve technique. Am. Midl. Nat., 87 (2) : 564-570.

Odum (H. T.). 1956. - Primary production in flowing waters. Limnol. Oceanogr., 1 : 102-117.

Odum (H. T.). 1957 a. - Trophic structure and productivity of silver springs, Florida. Ecol. Monogr., 271 : 55-112.

Odum (H. T.). 1957 b. -- Primary production measurements in eleven Florida springs and a marine Turtle-grass community. Limnol. Oceanogr., $2: 85-97$.

Owens (M.). 1965. - Some factors involved in the use of dissolved oxygen distributions in streams to determine productivity. $M e m$. Ist. Ital. Idrobiol., suppl. $18: 209-224$.

Owens (M.) et Maris (P. J.). 1964. - Some factors affecting the respiration of some aquatic plants. Hydrobiologia, $23: 533-543$.

Owexs (M.), Edwards (R. W.) et Gibbs (J. W.). 1964. - Some reaeration studies in streams. Int. J. Air Wat. Pollut., $8:$ 469-486.

WiNBERG (G. G.). 1971. - Methods for the estimation o: production of aquatic animals. Academic Press, London and New York, $175 \mathrm{p.}$ 